\title{
Appendix
}

HARD DATA

Real Personal Consumption Expenditures (SAAR, Bil.Chn.2009\$)

Disposable Personal Income (SAAR, Bil.\$)

Light Weight Vehicle Sales [Autos+Light Trucks] (SAAR, Mil.Units)

Mfrs' New Orders: Nondefense Capital Goods ex Aircraft (SA, Mil.\$)

Mfrs' Shipments: Nondefense Capital Goods ex Aircraft (SA, Mil.\$)

All Employees: Total Nonfarm (SA, Thous)

Agg Wkly Hrs Index: Prod \& Nonsupervisory: Total Pvt Industries (SA, 2002=100)

Civilian Unemployment Rate: $16 \mathrm{yr}+(\mathrm{SA}, \%)$

IP: Total Index (SA, 2012=100)

Housing Starts (SAAR, Thous.Units)

Private Construction: Residential (SAAR, Mil.\$)

Private Construction: Nonresidential (SAAR, Mil.\$)

Value of State and Local Construction Put in Place (SAAR, Mil.\$)

Exports: Goods and Services, BOP Basis (SA, Mil.\$)

Imports: Goods and Services, BOP Basis (SA, Mil.\$)

Inventory/Sales Ratio: Total Business (SA)
TRANSFORMATION

Delta log

Delta log

Log level

Delta log

Delta log

Delta log

Delta log

Log level

Delta log

Delta log

Delta log

Delta log

Delta log

Delta log

Delta log

Log level

\section{SOFT DATA}

TRANSFORMATION

Cass Freight Survey: Number of Shipments (WkDay Adj, Jan-90=1.000)

AIA: Architectural Billings Index (SA, $+50=$ Increasing)

Log level

ISM Mfg: PMI Composite Index (SA, 50+ = Econ Expand)

Log level

ISM Nonmanufacturing: NMI Composite Index (SA, 50+=Increasing)

Log level

Economic Policy Uncertainty Index (1985-09=100)

Log level

NFIB: Small Business Optimism Index (SA, 1986=100)

Log level

Conference Board: Consumer Confidence (SA, 1985=100)

Log level

University of Michigan: Consumer Sentiment (NSA, Q1-66=100)

Log level

Current Conditions: Buying Conditions for Large Household Goods (Relative

Score)

Log level

Home Builders: Housing Market Index [Composite] (SA, All Good = 100)

Log level

Fannie Mae Home Purchase Sentiment Index (NSA, Mar-11=60) - Seasonal

Adjustment, All

Log level

Wilshire 5000 Price Index [Full Cap] (AVG, Dec-31-70=830.27)

Log level

CBOE Market Volatility Index: VIX

Log level

Log level

NOTE: After transformation, all data series were demeaned and normalized by the sample standard deviation. All log transformations use natural logs. 